\title{
Low-frequency current fluctuations in doped ladders
}

\author{
Kenji Tsutsui $^{a}$, Didier Poilblanc ${ }^{b, a}$ and Sylvain Capponi ${ }^{a}$ \\ ${ }^{a}$ Laboratoire de Physique Quantique $\mathcal{E} 3$ UMR-CNRS 5626, Université Paul Sabatier, F-31062 Toulouse, France \\ ${ }^{b}$ Theoretische Physik, ETH-Hönggerberg, CH-8093 Zürich, Switzerland
}

(November 3, 2018)

\begin{abstract}
Charge current static and dynamical correlations are computed by exact diagonalisation methods on a 2-leg t-t'-J ladder which exhibits a sharp transition between a Luther-Emery (LE) phase of hole pairs and a phase with deconfined holes. In the LE phase, we find short-range low-energy incommensurate current fluctuations which are intrinsically connected to the internal charge dynamics within one hole pair. On the contrary, when holes unbind, the maximum of the current susceptibility moves abruptly to the commensurate wavevector $\pi$ and strongly increases for decreasing doping suggesting an instability towards a staggered flux state at sufficiently small doping.

PACS: 75.10.-b, 75.50.Ee, 71.27.+a, 75.40.Mg
\end{abstract}

Among mean-field solutions for two-dimensional (2D) strongly correlated electrons, the staggered flux phase [1] appeared as an appealing candidate. Due to the local $\mathrm{SU}(2)$ symmetry, the staggered flux phase is equivalent, at half-filling, to the projected d-wave pairing BCS variational wavefunction [2]. Both states could equivalently be viewed as a Resonating Valence Bond state [3]. Since hole doping lifts the degeneracy between the staggered flux state and the d-wave pairing state, it is of importance to investigate possible competition between such states. At finite doping, as the newly proposed d-density wave state [4], the staggered flux state would break translation as well as time reversal symmetry. In otherwords, it would exhibit "orbital antiferromagnetism" i.e. a checkerboard pattern of plaquette currents circulating clockwise and anti-clockwise on the two different sublattices. On the other hand, it has been shown [5] recently that the current vorticity correlations in a variational d-wave pairing state follows closely the hole correlations within one pair.

Numerical investigations carried out on small (2D) clusters have shown that staggered or commensurate (with a larger period modulation) flux states are very good variational states [6]. In addition, spin-chirality correlations have been tested numerically in the $2 \mathrm{D}$ tJ model [7] showing that staggered (i.e. at $\mathbf{q}=(\pi, \pi)$ ) chiral fluctuations are strongly enhanced with increasing hole kinetic energy. Recent calculations of the static current-current correlations on a 32-site lattice with two holes [8] have confirmed the existence of staggered vorticity in this model.

In this paper, search for current fluctuations have been carried out in an extended t-J model on a two-leg ladder where finite size effects can be handled in a controled fashion. Such a system is known to exhibit a robust spin gap at and close to half-filling as well as hole pair binding [9, 10]. Dominant power-law $\mathrm{d}_{x^{2}-y^{2}}$-like pairing and $4 k_{F}$ charge density wave (CDW) correlations at small doping are characteristic of a Luther-Emery (LE) liquid regime [11]. An additional negative diagonal hopping [12] $t^{\prime}$ between next-nearest neighbor (NNN) sites was shown to strongly reduce hole pair binding leading e.g. to the emergence of low-energy quasiparticle-like triplet excitations 13]. On the contrary, a positive $t^{\prime}$ increases the pair binding energy even further. Here, we use this parameter to monitor the hole pair binding energy in order to investigate possible connections between staggered-vorticity correlations and hole correlations. We find that incommensurate current fluctuations occur on the scale of the pair size. For sufficiently negative $t^{\prime}$ hole pairs break appart [13] and low energy staggered vorticity fluctuations are found as well as static commensurate correlations in the ground state (GS). We argue that these finding might signal an instability towards a staggered flux state.

The two-leg ladder Hamiltonian reads,

$$
\begin{aligned}
H & =J_{\text {rung }} \sum_{i}\left(\mathbf{S}_{i, 1} \cdot \mathbf{S}_{i, 2}-\frac{1}{4} n_{i, 1} n_{i, 2}\right) \\
& +J_{\operatorname{leg}} \sum_{i, l}\left(\mathbf{S}_{i, l} \cdot \mathbf{S}_{i+1, l}-\frac{1}{4} n_{i, l} n_{i+1, l}\right) \\
& +\sum_{i, j, l, m, \sigma} t_{i j}^{l m}\left(c_{i, l, \sigma}^{\dagger} c_{j, m, \sigma}+\text { h.c. }\right)
\end{aligned}
$$

where $i$ is a site index along the chain direction, $l$ and $m(=1,2)$ label the 2 legs and $n_{i, l}$ is the local charge density operator. Holes are assumed to hop along the chain direction or between legs with amplitudes $t_{i i+1}^{l l}=$ $t_{\text {leg }}$ and $t_{i i}^{12}=t_{i i}^{21}=t_{\text {rung }}$ respectively. In addition, as stated above, it is of particular interest to include a NNN hopping $t_{i \pm 1}^{12}=t_{i i \pm 1}^{21}=t^{\prime}$ [12]. In the following, for sake of simplicity, we shall only discuss the case of a spatially isotropic ladder i.e. with $t_{\mathrm{leg}}=t_{\text {rung }}=t$ (set to 1 ) and $J_{\text {leg }}=J_{\text {rung }}=J$. In addition, $J$ is set to 0.5. However, we believe our results are generic. The static and dynamical quantities reported in this paper have been obtained by exact diagonalisations (ED) of finite periodic $2 \times \mathrm{L}$ clusters ( $\mathrm{L}=8$ to $\mathrm{L}=12$ ) doped with up to 6 holes.

The NNN hopping $t^{\prime}$ is the key parameter of our study since it monitors the attraction between holes in the system [13]. To demonstrate this fact, as a preliminary study, we have computed the (static) charge correlations 
on 2 hole-doped ladders for many different parameters $t^{\prime}$. Selections of these data reported in Fig. 1 show clearly 2 different behaviors; above a critical value $t_{c}^{\prime}$ (we find $t_{c}^{\prime} \simeq-0.3$ at $J=0.5$ ) the two holes are bound and the hole-hole correlation decreases very fast with distance. Note that, in this case, finite size effects remain quite small since hole correlations at short distance are rather independent on system size. The bound pair "size" increases with decreasing $t^{\prime}$ and eventually at $t^{\prime}=t_{c}^{\prime}$ (negative) the GS changes abruptly 14 into a state where holes are unbound. Indeed, as shown for $t^{\prime}=-0.4$ in Fig. 1, the hole-hole correlation function increases with distance which means that holes tend to avoid each other. It is interesting to notice that boundary effects become quite important in this case probably in connection with the enhancement of the antiferromagnetic (AF) correlations. In order to avoid magnetic frustration, the 2 holes tend to be located, on average, on the same (opposite) leg for even (odd) ladder lengths 15. In the following, we shall show that the charge current fluctuations also change rapidly at the transition between bound pairs and individual holes.

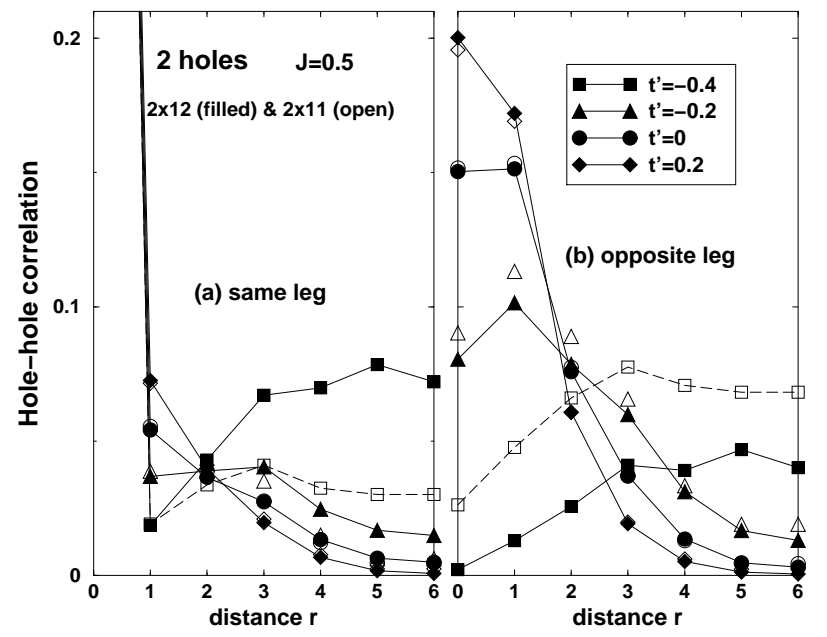

FIG. 1. Hole-hole (equal-time) correlation function on a two hole-doped $2 \times 12$ ladder vs separation between holes in the same leg (a) and in different legs (b). Different filled symbols correspond to different $t^{\prime}$ parameters according to the legend. Data obtained on a $2 \times 11$ ladder are also shown as open symbols (unless undistinguishable from the previous sets). Data are normalized to the hole density i.e. such that the local $(r=0)$ correlation equals 1 .

The current operators for the rung and leg bonds are defined as follows. For the leg,

$$
j_{q}=-i t_{\operatorname{leg}} \sum_{j, l, \sigma} e^{i(q j+\pi l)}\left(c_{j+1, l, \sigma}^{+} c_{j, l, \sigma}-h . c .\right),
$$

where $l$ and $n$ denote the site numbers for the leg and rung directions, respectively. Note that the leg current is defined in such a way that current densities on opposite bonds on the two legs have opposite signs as it should for loop currents defined on the plaquettes. For the rung,

$$
j_{q}=-i t_{\text {rung }} \sum_{j, l, \sigma} e^{i q j}\left(c_{j, l+1, \sigma}^{+} c_{j, l, \sigma}-h . c .\right) .
$$

In the staggered flux phase [1] both rung and leg currents would acquire a finite expectation value at momentum $q=\pi$ corresponding to a staggered arrangement of the loop currents on the plaquettes. Here we consider a more general situation of possible incommensurate modulations with arbitrary q [16] of the current pattern along the leg direction. Dynamical fluctuations of such loop currents can be investigated by computing the associated complex dynamical susceptibility,

$$
\chi_{j j}(q, \omega) \equiv \sum_{n} \frac{\left|\left\langle n\left|j_{q}\right| 0\right\rangle\right|^{2}}{\omega+i \epsilon+E_{n}-E_{0}},
$$

where $|n\rangle\left(E_{n}\right)$ are exact eigenstates (eigenenergies) of the hamiltonian. It is instructive to define the currentcurrent dynamical correlation function $S_{j j}$ as the imaginary part of the susceptibility devided by frequency,

$$
\begin{aligned}
S_{j j}(q, \omega) & \equiv \frac{\chi_{j j}(q, \omega)}{\omega} \\
& =\sum_{n} \frac{\left|\left\langle n\left|j_{q}\right| 0\right\rangle\right|^{2}}{E_{n}-E_{0}} \delta\left(\omega-E_{n}+E_{0}\right),
\end{aligned}
$$

which leads, after integration over frequency, to the static $(\omega=0)$ current susceptibility (Kramers-Krönig),

$$
\chi_{j j}(q, 0)=\int \frac{d \omega}{\omega} \chi_{j j}^{\prime \prime}(q, \omega)=\sum_{n} \frac{\left|\left\langle n\left|j_{q}\right| 0\right\rangle\right|^{2}}{E_{n}-E_{0}} .
$$

The current-current dynamical correlation $S_{j j}$ and the related static susceptibility have been computed on finite clusters by a standard continued-fraction method. Note that the susceptibility $\chi(q, 0)$ can alternatively be calculated as a response to a fictitious modulated magnetic flux. We have checked that this second method gives identical results. We also have found that, quite generally, use of bond and leg currents gives very similar results (especially at $q=\pi$ ) so that in the following, for sake of clarity, only results using the rung current operator will be presented.

We first focus on a ladder doped with only two holes. A selection of our results obtained on a $2 \times 12$ ladder are presented on Fig. 2. On should distinguish two interesting features on these data; (a) some high energy structure in the range $\omega \sim 4 t$ which is merely dispersing and whose weight increases with increasing $t^{\prime}$; (b) a low energy structure at a fraction of J. Above $t_{c}^{\prime} \simeq-0.3$, the maximum weight of this structure is spread on incommensurate 16] wavevectors in the vicinity of $\pi$. On the contrary, when the two holes become unbound $\left(t^{\prime}<t_{c}^{\prime}\right)$, 


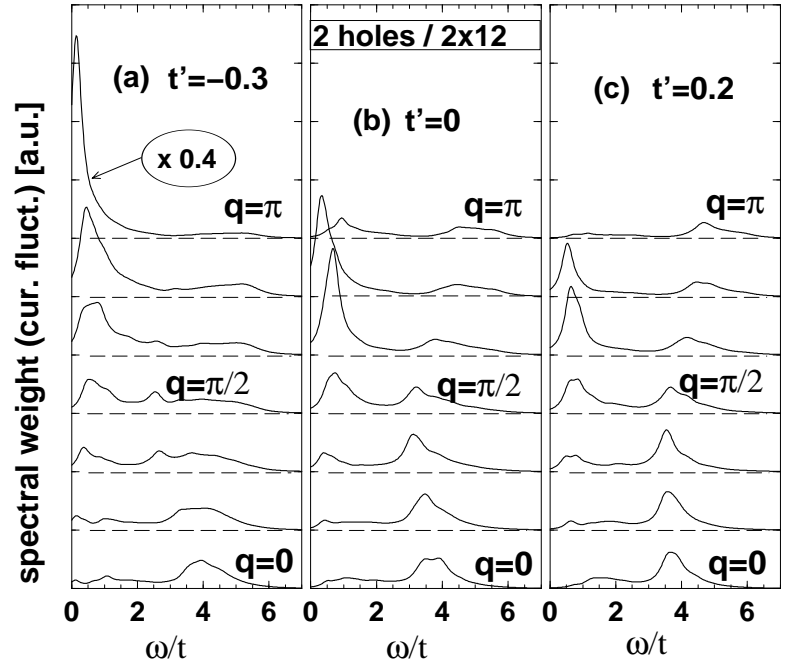

FIG. 2. Dynamical correlation function $S_{j j}(q, \omega)$ calculated on a 2 hole-doped periodic ladder for $J=0.5$. Each panel corresponds to a given value of $t^{\prime}$ as indicated. For clarity, curves obtained for all available momenta $q$ are shifted upwards w.r.t. each other by an equal amount. From bottom to top, $q=2 \pi n / L, n$ from 0 to $L / 2$. Note the reduction factor of 0.4 applied to the data at $t^{\prime}=-0.3$ and $q=\pi$.

the largest low energy (typically $\omega_{\text {typ }} \sim 0.2 J=0.1 t$ ) current fluctuations occur at $q=\pi$ corresponding to the staggered current pattern of the staggered flux state. The same behavior is also observed for higher doping as can be seen in Fig. 3 showing similar data but for a doping of four holes.

The behavior of the integrated spectral weight, namely the current-current susceptibility, as a function of parameters and doping is also of particular interest. Data reported in Fig. 1 indeed reveal that a qualitative change also occurs at the transition between bound hole pairs and free holes. When holes are tighly bound into pairs (typically for $t^{\prime}>0$ ) the current susceptibility is rather featureless and scales with the number of holes. When $t^{\prime} \rightarrow t_{c}^{\prime}$ from above, broad peaks appear in the vicinity of momentum $\pi$ [17] especially in the system containing only two holes. This increase of total spectral weight is mainly due to the increase of the low energy incommensurate peaks in the dynamical correlation functions shown in Figs. 2(b), 3(b). When hole pairs break apart i.e. for $t^{\prime}<t_{c}^{\prime}$ a large peak appears at the commensurate wavevector $q=\pi$.

To complete our study, we have also computed the equal-time current-current correlation function $\sum_{q} e^{i q r}\left\langle j_{q} j_{-q}\right\rangle$ vs separation $\mathrm{r}$ between two rungs. Recent Density Matrix Renormalisation Group (DMRG) studies on a 2 leg t-J ladder have shown that such correlations are exponentially suppressed with distance in the LE liquid phase [18]. Our data for various $t^{\prime}$ and 2 differ-

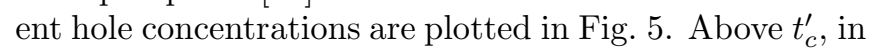

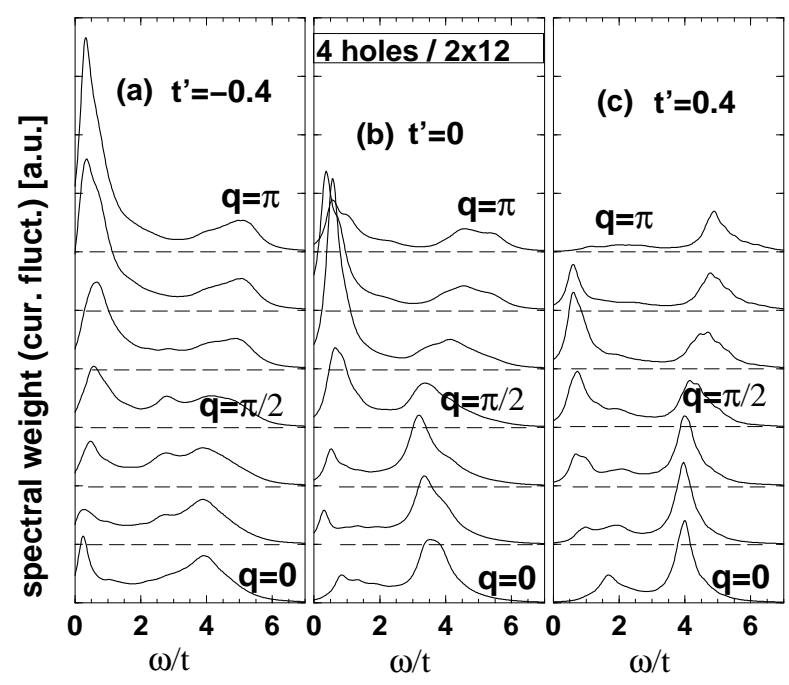

FIG. 3. Dynamical correlation function $S_{j j}(q, \omega)$ calculated on a 4 hole-doped periodic ladder for $J=0.5$. Same presentation as Fig. 目

the LE liquid phase, the alternating current correlations are indeed rapidly decreasing with distance in agreement with the DMRG results. When holes are strongly bound into pairs (as it is the case e.g. for $t^{\prime}=0.4$ ) it is interesting to notice that, apart from an overal scaling factor proportional to the hole concentration, the current correlation is almost completely independent of the number of pairs in the system. This is a further confirmation that current fluctuations occur within one pair. On the contrary, when pairs break apart the current correlations within the GS increase strongly [19] with decreasing hole density. In this case, establishment of staggered current correlations depends strongly on the parity of the ladder length because of possible frustration as seen in Fig. E)(a).

We finish this paper by briefly discussing the role of the SU(2) symmetry of the model. As mentioned above, it leads to the equivalence between $\mathrm{d}$-wave pairing and staggered flux phases at half-filling [2]. Since this degeneracy is lifted upon doping, the hole kinetic energy is expected to stabilize one phase over the other. The sudden change of properties in a t-t'-J ladder at small doping when varying $t^{\prime}$ is consistent with this scenario if one assumes that the competition between the pairing and the staggered flux phases is indeed governed by the small kinetic energy term (proportional to doping) and hence by $t^{\prime}$.

In summary, our data strongly suggest that, when hole pairs are stable, the current fluctuations is intrinsically related to the internal spin/charge dynamics within one pair at least in the regime where pairs do not overlap. The magnitude of the current fluctuations increases as the spatial extension of the pairs increases until they start to overlap. These findings are consistent with the picture of bound hole pairs of holes carrying opposite current 


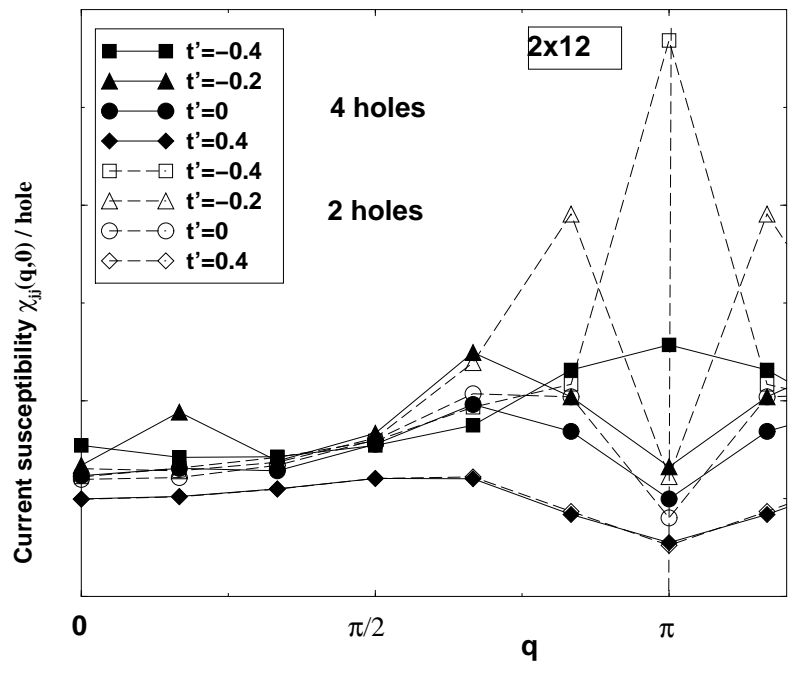

FIG. 4. Current-current susceptibility vs momentum q for different NNN hopping $t^{\prime}$ (as indicated on the plots) calculated on 2 and 4 -hole doped $2 \times 12$ ladders. The susceptibilities have been normalised w.r.t. the number of holes to emphasize proportionality if any. Arbitrary units are used. Note that at $t^{\prime}=0.4$ data for 2 and 4 holes are almost indistinguishable.

vorticity [5]. When hole pairs break apart into individual holes, commensurate staggered fluctuations set in. The increase of the magnitude of these fluctuations as one moves towards vanishing doping is interpreted as an instability to a (static) staggered flux state.

Computations were performed on the NEC-SX5 at IDRIS, Orsay (France). D.P. acknowledges support from the Center for Theoretical Studies and the Institute for Theoretical Physics at ETH-Zürich and thanks T.M. Rice for useful discussions.

[1] I. Affleck and J.B. Marston, Phys. Rev. B 37, 3774 (1988).

[2] F.C. Zhang, C. Gros, T.M. Rice and H. Shiba, Supercond. Sci. Technol. 1, 36 (1988).

[3] P.W. Anderson, Science 235, 1196 (1987).

[4] Sudip Chakravarty, R.B. Laughlin, Dirk K. Morr and Chetan Nayak, Phys. Rev. B 63094503 (2001).

[5] D.A. Ivanov, Patrick A. Lee, and Xiao-Gang Wen, Phys. Rev. Lett. 84, 3958 (2000).

[6] D. Poilblanc, Y. Hasegawa and T.M. Rice, Phys. Rev. B, 41, 1949 (1990); D. Poilblanc and Y. Hasegawa, Phys. Rev. B, 41, 6989 (1990).

[7] D. Poilblanc, E. Dagotto and J. Riera, Phys. Rev. B 43 7899 (1991).

[8] P.W. Leung, Phys. Rev. B 62, R6112 (2000).

[9] E. Dagotto and T.M. Rice, Science 271, 618 (1996); E. Dagotto, J. Riera, and D.J. Scalapino, Phys. Rev. B,

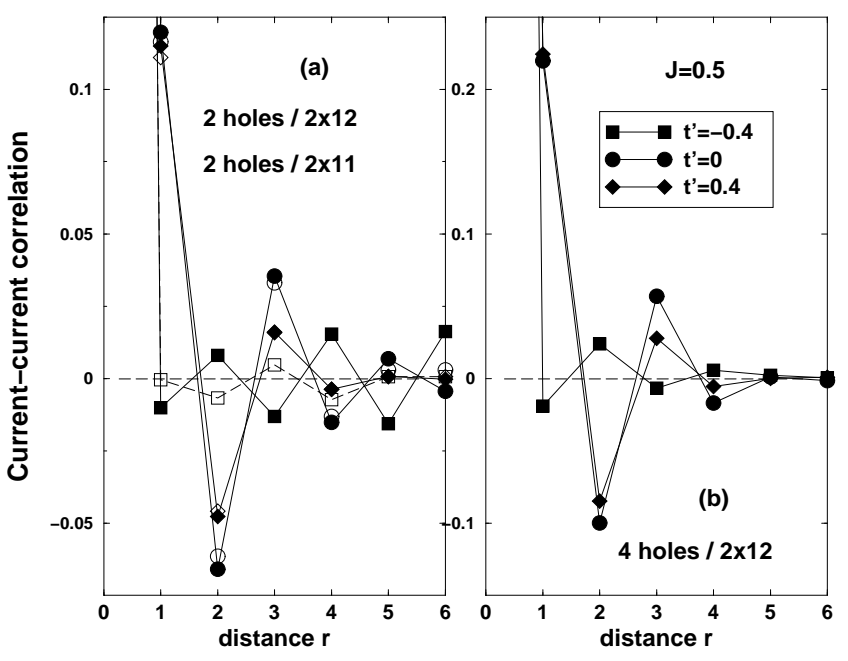

FIG. 5. Current (equal-time) correlation function vs distance for two (a) and four (b) hole-doped ladders calculated on a $2 \times 12$ cluster. Different filled symbols correspond to different $t^{\prime}$ parameters according to the legend. In (a) data for a $2 \times 11$ ladder are also shown as open symbols (unless undistinguishable from the previous sets).

45, 5744 (1992); T.M. Rice, S. Gopalan, and M. Sigrist, Europhys. Lett. 23, 445 (1993).

[10] C. A. Hayward et al., Phys. Rev. Lett. 75, 926 (1995).

[11] D. Poilblanc, D.J. Scalapino, and W. Hanke, Phys. Rev. B, 52, 6796 (1995); M. Troyer, H. Tsunetsugu, and T.M. Rice, Phys. Rev. B, 53, 251 (1996); C.A. Hayward and D. Poilblanc, Phys. Rev. B, 53, 11721 (1996).

[12] $t^{\prime} / t \simeq-0.35$ has been suggested for the $2 \mathrm{D}$ high- $\mathrm{T}_{C}$ cuprates. See e.g. C. Kim et al., Phys. Rev. Lett. 80, 425 (1998).

[13] D. Poilblanc et al., Phys. Rev. B, 62, 14633 (2000).

[14] Note that in most cases (e.g. 2 holes on $2 \times 10$ and $2 \times 12$ lattices, 4 holes on $2 \times 12$, etc...) this transition is linked to a level crossing between two GS wavefunctions with different total momenta.

[15] For details on the role of the parity of the ladder length see e.g. J. Riera, D. Poilblanc and E. Dagotto, Eur. Phys. J. B 7, 53 (1999).

[16] By "incommensurate" we, in fact, mean commensurate modulations with larger periodicities.

[17] Note that the static charge structure factor exhibits a sharp " $4 \mathrm{k}_{F}$ " singularity at $q=2 \pi n$ for $t^{\prime} \geq 0$ as expected in a LE liquid. This feature tends to quickly deasappear for increasingly negative $t^{\prime}$. Details will be reported elsewhere.

[18] D.J. Scalapino, S.R. White and I. Affleck, "Rung-Rung Current Correlations on a 2-Leg t-J Ladder", condmat/0102182.

[19] Increase of equal-time current-current correlations under the effect of an extra short range Coulomb repulsion have also been reported; see J.B. Marston and Asle Sudbø, "Staggered Orbital Currents in a Model of Strongly Correlated Electrons", cond-mat/0103120. 